\title{
POTENCIALES EVOCADOS VISUALES EN LAA ENFERMEDAD DE CHAGAS-MAZZA CRONICA
}

\author{
RAUL F. PELLI-NOBLE * OSCAR D. IGUZQUIZA * \\ ESTELA GRAIF'F DE RIARTE *
}

RESUMEN - Se efectuaron potenciales evocados visuales (PEV) en sus variantes flash (F) y pattern (P), en 17 pacientes afectados de enfermedad de Chagas crónica. La edad del grupo se halló comprendida entre $\operatorname{los} 21$ y 65 años. Se incluyeron pacientes con periodo de evolución mayor de 7 años y dos serologias positivas como mínimo. La selección fue descartando a los portadores de diabetes, alcoholismo, lepra, sifilis y enfermedades degenerativas del sistema nervioso central, como asf intoxicaciones de diversas etiologias y afecciones visuales detectadas en el exámen oftalmológico. Todos fueron estudiados con exámenes clínicos y complementarios de rutina, electrocardiograma, exámen oftalmológico y específicos como la reacción de Machado-Guerreiro, test de inmunofluorescência $y$ test de hemoaglutinación. Los resultados de los PEV mostraron fundamentalmente alteraciones en la morfologia del trazado $\mathrm{y}$ disminución de la amplitud del potencial, en el $35 \%$ de los pacientes estudiados. Estas alteraciones electroneurofisiológicas permitirían inferir correlación a los hallazgos anatomopatológicos, que muestran pérdida de grupos neuronales en autopslas de pacientes chagésicos crónicos.

\section{Visual evoked potentials in chronic Chugas-Mazza disease.}

SUMMARY - Both modalities of visual evoked potentials (VEP), flash (F) and pattern $(P)$, were carried out in 17 patients with chronic Chagas disease. The patients included, between 21 and 65 years old, presented an evolution period of more than 7 years and a minimum of two positive serologles. Those patients with diabetes, alcoholism, leprosy, syphilis and degenerative diseases of the central nervous system, so as intoxications of different etiologfes, and visual disorders detected through ophthalmological examination were discarded. Everyone was studied by means of routine clinical and complementary analysis, electrocardiogram, ophthalmological examination and specific analyses for the disease, like Machado-Guerreiro reaction, immunofluorescence and hemoggolutination tests. The VEP results showed alterations in the morphology of the record and a decrease of the potential amplitude in $35 \%$ of these patients. The electroncurophysiological alterations would suggest a correlation with anatomopathologic findings, that show loss of neuronal groups in autopsies of chronic Chagasic patients.

La tripanosomiasis americana o enfermedad de Chagas-Mazza es una de las principales endemias de los países latinoamericanos. Se extiende desde el sur de los Estados Unidos de Norteamérica hasta la provincia de Chubut en la República Argentina. El ataque del Trypanosoma cruzi al organismo es multivisceral, siendo los más afectados los aparatos cardiovascular, digestivo y el sistema nervioso. Ello llevaría a una alteración funcional y aparición de patologías clínicas en los sectores agredidos. Las alteraciones neurológicas en el período crónico se refieren a sindrome de neurona

* Instituto de Binelectrónica de la Universidad Nacional de Tucumán, UNT (Director: Prof. Dr. Fernando Martínez Corvalán). 
periférica, polineuritis, radiculitis con hipo o arreflexia, trastornos del lenguaje, hipercinesia, síndrome seudobulbar, trastornos psiquiátricos y todos aquellos que corresponden a lesiones de cordones posteriores a la médula 1,6,9,22,23-25. Además, se menciona la existencia de un sindrome neurológico mínimo (SNM), que se exterioriza por cetaleas, astenia, constipación, mareos y somnolencia 14 .

El objetivo de este estudio es la aplicación de los potenciales evocados visuales (PEV) en sus variantes flash (F) y pattern (P), como técnica de estudio para objetivar las alteraciones funcionales, aún las asintomáticas y subclínicas de la via visual en el período crónico de la enfermedad de Chagas 2,7,8,15,18-21,26. Las mismas podrían relacinarse con las lesiones que en el SNC provoca esta enfermedad en su etapa crónica y que fueron demonstradas en estudios anatomopatológicos 4,5,10-13.

\section{MATERIALES Y METODO}

Se seleccionaron 17 pacientes afectados de enfermedad de Chagas-Mazza crónica. La edad del grupo se ubicó entre los 21 y 65 años con distribución por sexo del $58,8 \%$ de hombres $y$ el $41,2 \%$ de mujeres. El tiempo de evolución de la enfermedad se ubicó entre los 8 y 35 años. El 24,3\% describió el momento de contagio manifestando el chagoma de inoculación o complejo oftalmoganglionar. En los demás se tomó como punto de referencia el tiempo transcurrido desde el primer exámen serológico positivo. Siendo requisito indispensable para los pacientes a ser incluídos en el grupu, dos serologías positivas como minimo. Se descartaron aquellos que presentaron diabetes, alcoholismo, lepra, sililis y otras enfermedades degenerativas del sistema nervioso central como así intoxicaciones de diversas
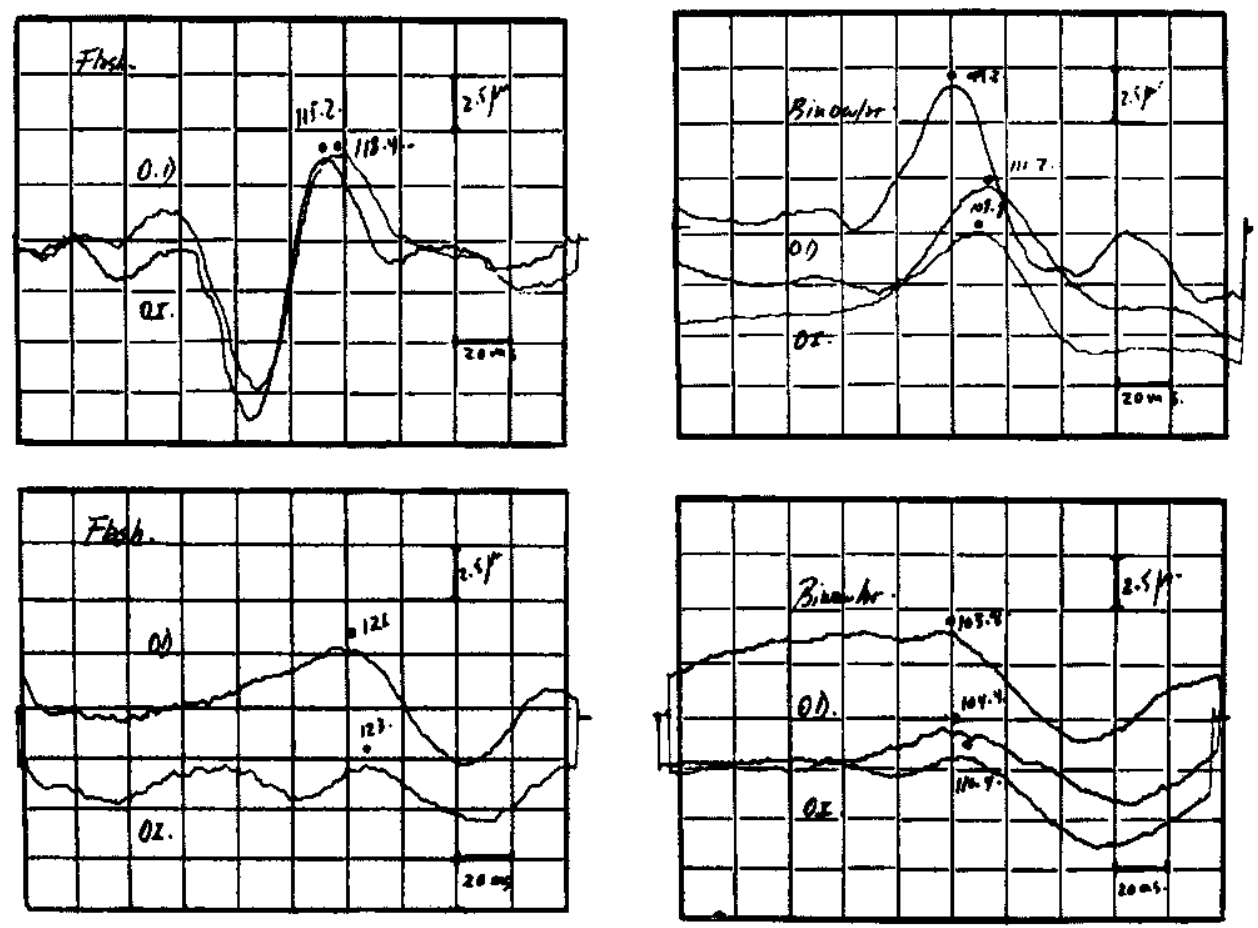

Fig. 1 - Izquienda: PEV Flash - trazado superior: onda IV de Ciganek de conformación $y$ latencia normales; trazado inferior: alteraciones en morfología y amplitud de la onda IV de Ciganek (Derivacion $F_{z} P_{z}$; promediado de 100 respuestas). Derecha: $P E V$ Pattern trazado superior: componente $P_{100}$ de caracteristicas normales; estimulaci6n a campo completo binocular $y$ monocular alternado; trazado inferior: morfologia alterada y amplitud disminuida del componente $P_{100}$ (Derivacion sagital $F_{z} P_{z}$; promediado de 100 respuestas). 
etiologias $\mathrm{v}$ afecciones visuales detectadas en el exámen oftalmológico. Todos fueron evaluados con exámen clínico, electrocardiograma, exámen oftalmológico, análisis químicos de rutina y Jas reacciones de Machado-Guerreiro, hemoaglutinación e inmunofluorescencia.

Los PEV fueron efectuados en sus modalidades $F^{\prime}$ y $P$. Fue utilizada la derivacion sagital $\mathrm{F}_{z} \mathrm{P}_{\mathrm{z}}$ de la clasificación internacional, con registros monoculares derecho $e$ izquierdo $y$ binocular. Se promediaron 100 estímulos de una duración de 0,2 mseg a una frecuencia de 2 estímulos por segundo. Tiempo de análisis de $200 \mathrm{mseg}$ de latencia con filtros de alta y baja de 100 y $1 \mathrm{~Hz}$ respectivamente.

\section{RESULTADOS}

De los 17 pacientes estudiados, el 65\%, 11 pacientes, presentaron PEV normales. Los 6 pacientes restantes (35\%) presentaron PEV alterados en morfología y amplitud. El PEV en su variante $\mathbf{P}$ o damero luminoso reversible, mostró alteraciones en los 6 pacientes; la modalidad $F$ en 5. En el PEV modalidad P, la morfología del componente P100 se presentó alterada en 1 paciente $(5,8 \%)$. En la variedad $\mathbf{F}$ en cambio, la morfología de la onda IV de Ciganek presentó alteraciones en 4 casos $(17,6 \%)$. La disminución de la amplitud fue la alteración más significativa de los PEV. La misma se ubicó entre 1,25 y 3 uV. En la modalidad $P$ en 5 pacientes $(29,3 \%)$ y en la modalidad $F$ en 3 casos $(17,6 \%)$ (Tabla 1). La figura 1 permite apreciar las alteraciones mencionadas.

De acuerdo a las manifestaciones neurologicas, los pacientes fueron incluídos en tres grupos (Tablas 2 y 3 ):

Grupo A - Con alteraciones neurológicas como hiporreflexia osteotendinosa, hipotonía, disminución de la fuerza muscular a predominio de miembros inferiores. Manifestaciones subjetívas de alteraciones visuales como disminución de la agudeza visual y fotógenos. Fueron incluidos 4 pacientes PEV alterado en uno de ellos.

Grupo B - Con sintomas de un SNM conformado por cefaleas, astenia, adinamia, mareos y somnolencla. Se incluyeron 5 pacientes de los cuales 4 presentaron PEV alterados en sus dos modalidades $F$ y $\mathbf{P}$.

Grupo C - Exámen clínico-neurológico y oftalmológico normales. De los 8 pacientes incluidos sólo 1 presento alteraciones en el PEV en sus dos modalidades F y P.

\begin{tabular}{|c|c|c|c|c|c|c|c|c|c|c|c|c|c|c|c|}
\hline \multirow{3}{*}{$\begin{array}{c}N^{\circ} \\
\text { Hist. } \\
\text { clinn. }\end{array}$} & \multicolumn{3}{|c|}{ PEV $P$ binocular } & \multicolumn{6}{|c|}{ PEV $P$ monocular } & \multicolumn{6}{|c|}{ PEY $F$} \\
\hline & & & & & & & & \multicolumn{2}{|l|}{ M } & & & & & \multicolumn{2}{|c|}{ M } \\
\hline & & & & 0.0 & 0.1 & 0.0 & 0.1 & 0.0. & 0.1 . & 0.0 & 0.1 . & 0.0 & 0.1 & 0.D. & 0.1 . \\
\hline 001 & 1.25 & 105.6 & $\mathrm{~N}$ & 1.25 & 1.25 & 111.2 & 112.8 & A & A & 7.5 & 7.5 & 110. & 112.8 & $\mathbf{N}$ & $\mathbf{N}$ \\
\hline 002 & 3 & 104. & $\mathbf{H}$ & 2.5 & 2.5 & 110.4 & 112 & N & $\mathrm{N}$ & 1.25 & 2.5 & 119.2 & 120 & $N$ & $\mathbf{N}$ \\
\hline 003 & 2.5 & 99.2 & $\mathrm{~N}$ & 2. & 2. & 102. & 104.8 & N & $\mathrm{N}$ & 2.5 & 2.5 & 112.8 & 111.2 & A & A \\
\hline 004 & 3. & 103.8 & A & 2. & 2. & 104.4 & 110.4 & $A$ & $A$ & 2. & 3. & 135. & 138.4 & A & A \\
\hline 005 & 2.5 & 107.2 & $\mathbf{N}$ & 2.5 & 1.25 & 106.4 & 108.8 & $\mathrm{~N}$ & $\mathrm{~N}$ & 10. & 10. & 118. & 122. & A & A \\
\hline 006 & 10. & 102. & $\mathbf{N}$ & 5. & 5. & 107. & 105. & $\mathrm{~N}$ & $\mathrm{~N}$ & 10. & 10. & 105. & 110. & $\mathbf{N}$ & $\mathbf{N}$ \\
\hline 007 & 10. & 103.6 & $\mathrm{~N}$ & 7.5 & 7.5 & 106. & 105. & N & $\mathrm{N}$ & 10. & 10. & 115.2 & 118. & $\mathbf{N}$ & $\mathbf{N}$ \\
\hline 008 & 15. & 100.2 & $\mathrm{~N}$ & 10. & 10. & 109.2 & 107. & $\mathrm{~N}$ & $\mathrm{~N}$ & 10. & 15. & 112. & 115. & $\mathbf{N}$ & $\mathbf{N}$ \\
\hline 009 & 15. & 99.2 & $\mathrm{~N}$ & 10. & 10. & 111.2 & 108.8 & $\mathrm{~N}$ & $\mathrm{~N}$ & 15. & 15. & 122 & 128. & $\mathbf{N}$ & $\mathbf{K}$ \\
\hline 010 & 10. & 113.6 & $\mathrm{~N}$ & 7.5 & 7.5 & 108. & 107.2 & $N$ & $N$ & 10. & 10. & 115.2 & 118.4 & $\mathrm{~N}$ & $H$ \\
\hline 011 & 10. & 103. & $\mathrm{~N}$ & 5. & 5. & 105. & 108. & $N$ & $\bar{N}$ & 10. & 10. & 120. & 118. & $N$ & $\mathrm{~N}$ \\
\hline 012 & 10. & 100. & $\mathrm{~N}$ & 7.5 & 7.5 & 100. & 100 & $N$ & N & 10. & 10. & 135. & 133.6 & A & A \\
\hline 013 & 10. & 100. & $\mathrm{~N}$ & 5. & 5. & 102. & 104. & $N$ & $\mathrm{~N}$ & 10. & 10. & 120. & 118. & $N$ & N \\
\hline 014 & 7.5 & 102 & $\mathrm{~N}$ & 5. & 5. & 105. & 107. & $\mathrm{~N}$ & $N$ & 15. & 15. & 115. & 117. & $N$ & $x$ \\
\hline 015 & 10. & 105. & $\mathrm{~N}$ & 5. & 5. & 108. & 110. & $N$ & $N$ & 10. & 10. & 122. & 127. & N & $\mathrm{H}$ \\
\hline 016 & 10. & 103. & $\mathrm{i}$ & 7.5 & 7.5 & 102. & 105. & $N$ & $N$ & 10. & 10. & 102. & 112. & A & $\mathrm{H}$ \\
\hline 017 & 10. & 100. & $\mathbf{N}$ & 7.5 & 7.5 & 105. & 108. & $N$ & N & 10. & 10. & 112. & 115. & N & $K$ \\
\hline V.N. & $10 \pm 5$ & $110 \pm 2$ & - & $10 \pm 5$ & $10 \pm 5$ & $110 \pm 2$ & $110 \pm 2$ & - & - & $10 \pm 5$ & $10 \div 5$ & $110 \pm 2$ & $110 \pm 2$ & - & - \\
\hline
\end{tabular}

Tabla 1 - Resultiados obtenidos en el PEV flash y pattern. $M$, morfologia; $N$, normal; $A$, alterada. Valores normales: amplitud $10 \pm 5$; latencia $110 \pm 2$. 


\begin{tabular}{|c|c|c|c|c|c|c|}
\hline \multicolumn{3}{|c|}{ Grupos } & No Pac & PEV Alt & \multicolumn{2}{|l|}{$\%$} \\
\hline \multicolumn{3}{|c|}{ Alt neurológicas } & 2 & 1 & \\
\hline \multicolumn{3}{|c|}{ Alt visuales subjetivas } & 2 & - & \multicolumn{2}{|l|}{$1 / 4=25 \%$} \\
\hline \multicolumn{3}{|c|}{ B SNM } & $\mathbf{5}$ & 4 & \multicolumn{2}{|l|}{$4 / 5=80 \%$} \\
\hline \multicolumn{3}{|c|}{ C Normal } & 8 & 1 & \multicolumn{2}{|l|}{$1 / 8=12.5 \%$} \\
\hline \multicolumn{3}{|c|}{ Total Pac } & 17 & 6 & \multicolumn{2}{|l|}{$6 / 17=35 \%$} \\
\hline \multicolumn{7}{|c|}{$\begin{array}{l}\text { Tabla \& - Clasificacion y resultados } \\
\quad \text { sindrome neurologico mímimo. }\end{array}$} \\
\hline Sintomas & y Signos & No de Casos & Síntomas & y Signos & & Ne de Casos \\
\hline \multicolumn{3}{|c|}{ Astenia * $\quad 10$} & \multicolumn{4}{|c|}{ Hipersomnia* } \\
\hline \multicolumn{2}{|l|}{ Mialgias } & 7 & \multicolumn{4}{|c|}{ Trastonos de memoria * } \\
\hline \multicolumn{2}{|c|}{ Adinamia * } & 7 & \multicolumn{4}{|c|}{ Trastornos visulales subjetivos } \\
\hline \multicolumn{2}{|c|}{ Parestesias } & 5 & \multicolumn{3}{|c|}{ (fotógenos, disminución agudeza visual) } & 2 \\
\hline \multicolumn{2}{|c|}{ Constipacion } & 4 & \multicolumn{3}{|c|}{ Hiporreflexia osteotendinosa } & 2 \\
\hline \multicolumn{2}{|l|}{ Neuralgias } & 3 & \multicolumn{3}{|c|}{ Disminución del tono muscular } & 2 \\
\hline \multicolumn{2}{|l|}{ Cefaleas * } & 3 & \multicolumn{3}{|c|}{$\begin{array}{l}\text { Disminución de la fuerza muscular } \\
\text { Mareos } *\end{array}$} & $\begin{array}{l}2 \\
1\end{array}$ \\
\hline
\end{tabular}

Tabla 3 - Manifestaciones clinicas destacadas. (*), corresponden a un SNM.

\section{COMENTARIOS}

La forma neurológica de la enfermedad de Chagas-Mazza crónica constituye entidad nosológica de difícil caracterización clinica. Pues en la mayoría de los casos los exámenes neurológicos son normles o muestran sólo leves alteraciones. Su diagnóstico se basa sobre todo en el antecedente epidemiológico y las reacciones serológicas positivas en pacientes con alteraciones neurológicas mínimas, incluyendo el SNM. Mazza y Jorg señalaban la importancia de buscár sistemáticamente en los pacientes provenientes de zonas endémicas, las «expresiones minimas», como llamaban al SNM 16. En nuestro estudio, consideramos como hecho significativo el hallazgo de alteraciones en el PEV de 4 de los 5 pacientes que presentaban clínicamente sólo un SNM.

De todos los pacientes estudiados, el 35\% presentó alteraciones en el PEV. Gráficamente, la modificación más evidente fue la disminución de la amplitud de la onda IV de Ciganek y del componente P100, que osciló entre 1,25 y 3 uV, en ambas modalidades.

Los expuesto en el presente estudio permite sugerir que las alteraciones halladas en el PEV de pacientes chagásicos crónicos serian debidas a la disfunción o reducción del número de neuronas activas que responden sincrónicamente al estimulo aplicado, hecho ya demonstrado en estudios anatomopatológicos 4,5,10-13, no descartándose la posible existencia de trastornos a nivel sináptico. Se muestra tambien a los potenciales evocados como un método objetivo adecuado para detectar las alteraciones de la enfermedad de Chagas-Mazza crónica, aún las mínimas, como lo indica el alto porcentaje de alteraciones del PEV en pacientes con SNM.

Agradecimiento - Los autores agradecen a los Doctores Jullo Ousset, Jefe del Servicio Nacional de Chagas Jurisdicción Regional, Carlos E. Silva y Hugo Norry, quienes proporcionaron gran parte del material de pacientes con que fue efectuado este estudio. Asimismo, a los Doctores María Cristina Garcia Mena de Alabarse, Luis Alberto Calderó y Susana Yolanda Novoa por su colaboración en el mismo. Al Profegor Doctor Fernando Martínez Corvalán, agradecemos au colaboración en los comentarios y correación del trabajo. Este trabajo fue realizado merced a los aportes de: Rectorado de la Universidad Nacional de 
Tucumán, Consejo de Investigaciones de la Universidad Nacional de Tucumán y Programa Nacional de Informática y Electrónica de la Secretaría de Ciencia y Técnica del Ministerio de Educación $y$ Justicia de la Nación.

\section{REFTRENCIAS}

1. Armbrust-Figueiredo $J$, Jardim $E$ - Diagnosis of the chronic neurological form of Chagas disease, X Inter Congr Neurol. Excerpta Médica 296:11, 1973.

2. Bogacz JJ, Barajas J, Celesia G, Cracco J, Desmedt J, Garcia H, Helcox K, Peters J, Sormer H, Kenneth S - Los Potenciales Evocados en el Hombre. Ateneo, Buenos Aires, 1985, pg 57.

3. Bonet AH - Epidemiología de la enfermedad de Chagas en la República Argentina. Simposio Internac Enfermedad de Chagas. Buenos Aires, 1972.

1. Brandåo $\mathbf{H J}$, Zulian $\mathbf{R}$ - Nerve cell depopulation in chronic Chagas disease: a quantitative study in the cerebellum. Rev Inst Med Trop São Paulo 8:281, 1966.

5. Britto-Costa R, Gallina RA - Hivotálamo anterior na moléstia de Chagas humana. Rev Inst Med Trop Sáo Paulo 13:92, 1971.

6. Chagas C - Les formes nerveuses d'une nouvelle trypanosomiase (T. cruzd) inocule par Triatoma megista (Maladie de Chagas). Nouv Iconogr Salpetrière 26:1, 1913.

7. Desmedt JE - Cerebral evoked potentials. In Dyck PJ, Thomas PK, Lambert EH, Bunge R (eds): Peripheral Neuropathy, Vol 1. Ed 2. Saunders, Philadelphia, 1984, pg 1045 .

8. Desmedt JE, Noel P - Averaged cerebral evoked potentials in the evaluation of lesions of the sensury nerves and of the central somatosensory pathway. In Desmedt JE (ed): New Developments in Electromyography and Clinical Neurophysiology, Vol 2. Karger, Basel, 1973, pg 352.

9. Forjaz SV - Aspectos neurológicos da doença de Chagas: sistema nervoso central Arq Neuro-Psiquiat (Sāo Paulo) 25:175, 1967.

10. Koberle $\mathbf{F}$ - Patología y anatomía patologica de la enfermedad de Chagas. Bol of Sanit Panamer 51:404, 1962.

11. Koberle $\mathbf{F} \rightarrow$ Chagas disease and Chagas syndrome: the pathology of American trypanosomiasis. Adv Parasitol 6:63, 1968.

12. Koberle F $\rightarrow$ The causation and importance of nervous lesions in American trypanosomiasis. Bull Wld Hlyh Arg 42:739, 1970.

13. Koberle F, Alcantara FG - Mecanismo da destruição neuronal do sistema nervaso periférico na moléstia de Chagas. Hospital 57:173, 1960.

14. Libonatti E - Formas neurológicas de la enfermedad de Chagas. Cerebrum 1:41, 1983.

15. Manguiere F, Fischer C - Les potentiels evoqués dans les affections neurologiques. Encyplopédie Médico-Chirurgicale: Neurologie 170 31: B10. Masson, Paris, 1982, pg 1.

16. Mazza S, Jorg ME - Períodos anátomo-clínicos de la enfermedad de Chagas. Prensa Méd Arg 27:2361, 1940.

17. Pagano MA, Basso S, Sica REP, Aristimuño GG, Colombi A - Electromyographical findings in human chronic Chaga's disease. Arq Neuro-Psiquiat (São Paulo) 36:316. 1978.

18. Pelli Noble RF, Carreño Rimaudo S, Martinez Corvalan F, Herrero A, Campero RM Fundamentos del Registro de los Potenciales Evocados en la Neuropatía Chagásica. Soc Arg Bioingeniería (SABI'83). Residencia Universitaria FIorco Molle, Tucumán, 1983.

19. Pelli Noble RF, Martinez Corvalan F, Carreño Rimaudo S, Herrero A, Campero RF Fundamentos actuales en potenciales evocados. Rev Neurol Arg 2:239. 1985.

20. Pelli Noble RF, Carreño Rimaudo S, Caldero LA $\rightarrow$ Aplicaciones clínicas de potenciales evocados sensoriales y congnitivos en la práctica médica. Rev Fac Med UNT 20 (2), 1988.

21. Pelli Noble RF, Caldero LA, Novoa S, Iguzquiza O - Respuestas evocadas visuales en la tripanosomiasis americana. XXVIII Congr Arg Neurol, Mendoza, 1988.

22. Sanz OP - Compromiso de la neurona del asta anterior de la médula en la enfermedad de Chagas crónica. Rev Neurol Arg 3:458, 1979.

23. Sanz O, Aristimuño GG, Sica REP, Ratusnu AF, O'Neill EM - An electrophysiologica] investigation of skeletal muscle in human chronic Chagas' disease. Arq Neuro-Psiquiat (Săo Paulo) 36:319, 1978.

24. Sanz OP, Sica REP, Fumo $T$, Basso $S$ - Spinal reflex activity in patients with chronic Chagas' disease. Medicina (Buenos Aires) 42:633, 1982.

25. Sica REP, Filipini D, Panizza M, Fumo $T$, Basso S, Lazzari J, Molina HA - Involvement of the peripheral sensory nervous system in human chronic Chagas disease. Medicina (Blienos Aires) 46:662, 1986.

26. Spelreijse H, Estevez O, Reits D - Visual evoked potentials and physiological analysis of visual processes in man. In Desmedt JE (ed): Visual Evoked Patentials in Man: New Developments. Oxford, 1977. 